\title{
Recent developments in $U(3) \chi$ PT: meson-meson scattering and finite energy sum rules
}

\author{
Zhi-Hui Guo* \\ Department of Physics, Hebei Normal University, 050024 Shijiazhuang, P. R. China \\ and Departamento de Física, Universidad de Murcia, E-30071 Murcia, Spain. \\ E-mail: guodum.es
}

\section{J.A. Oller}

Departamento de Física, Universidad de Murcia, E-30071 Murcia, Spain.

E-mail: Qlerdum.es

\section{J. Ruiz de Elvira}

Departamento de Física Teórica II, Universidad Complutense de Madrid, E-28040 Madrid, Spain.

E-mail: Gacoboredrect.ucm.es

\begin{abstract}
We discuss the meson-meson scattering and finite energy sum rule (FESR), based on the one-loop calculation within $U(3)$ chiral perturbation theory $(\chi \mathrm{PT})$. First we obtain the pertinent resonance spectroscopy from the unitarized partial wave scattering amplitudes. Then we investigate how well the FESR can be satisfied in the physical situation at $N_{C}=3$. Further discussions on the extrapolation of $N_{C}$ are also given.
\end{abstract}

Sixth International Conference on Quarks and Nuclear Physics,

April 16-20, 2012

Ecole Polytechnique, Palaiseau, Paris

${ }^{*}$ Speaker. 


\section{Introduction}

Scalar dynamics of the light quark flavors in the nonperturbative energy region (especially below $1 \mathrm{GeV}$ ) has been quite a topical research subject in the last decade, see Refs. [(⿴囗⿰丿㇄口, 口, [1, 团]. Despite the confirmation of the mass and width of the broad resonances by different groups [[]], their nature is still under a vivid debate. In the recent works [ [ [ [], we make a comprehensive study of the scalar resonances below $1.5 \mathrm{GeV}$. Here we mainly discuss the results on the meson-meson scattering and FESR (a way to quantify the semi-local or average duality) [6].

First, the meson-meson scattering amplitudes are unitarized to fit to experimental data and the relevant resonance spectroscopy is confirmed. After then, we further investigate the role of resonances in semi-local duality, not only for the physical case at $N_{C}=3$, but also for the situation with $N_{C}>3$. The fulfillment of the nontrivial aspects of finite energy sum rules poses a strong support of the proposed picture for the scalar dynamics in our work.

\section{Theoretical framework}

Our theoretical framework is $U(3) \chi \mathrm{PT}[\square$, [], which includes not only the pseudo-Goldstone octet $\pi, K$ and $\eta_{8}$, as $S U$ (3) $\chi \mathrm{PT}$ [9], but also the singlet $\eta_{1}$. After the diagonalization of the $\eta_{8}-\eta_{1}$ mixing, one has the physical $\eta$ and $\eta^{\prime}$ mesons as the dynamical degrees of freedom in $U(3) \chi \mathrm{PT}$. This qualifies $U(3) \chi \mathrm{PT}$ as an improved approach for studying the $\eta$ and $\eta^{\prime}$ dynamics compared to the $S U(3)$ version. Instead of considering the contributions from the higher order low energy constants (LECs), we introduce explicitly the resonance exchanges at tree level, assuming the resonance saturation of LECs in our work. We follow the resonance chiral theory [एँ] to include the scalar, vector and pseudoscalar resonance contributions. In addition we take into account another two local chiral operators $\delta L_{8}$ and $\Lambda_{2}$ in the calculation [四, 口].

We calculate the mass and wave function renormalizations, pion decay constant and all of the meson-meson scattering processes in $U(3) \chi \mathrm{PT}$ up to one-loop order plus the tree level exchange of resonances. The perturbative amplitudes with definite isospin $I$ and angular momentum $J$ are then unitarized through the nonperturbative N/D approach [团]

$$
T^{I J}(s)=\left[1+N^{I J}(s) g^{I J}(s)\right]^{-1} N^{I J}(s),
$$

where $g^{I J}(s)$ takes care of the nonperturbative resummation of the unitarity cuts and all the contributions from the crossed-channel cuts are collected in $N^{I J}(s)$. The explicit forms for $g^{I J}(s)$ and $N^{I J}(s)$ are given in Ref. [四]. With the unitarized partial waves in Eq. (․]. to calculate the phase shift, inelasticity and invariant mass distribution, which can be used to fit experimental data.

In addition to the phenomenological aspects, there also exist theoretical constraints on the scattering amplitudes. We focus on the semi-local duality in $\pi \pi$ scattering between the Regge theory and hadronic degrees of freedom in this work. One of the objects to quantify the semi-local (or average) duality is the FESR

$$
\int_{v_{1}}^{v_{2}} v^{-n} \operatorname{Im} T_{\mathrm{t}, \text { Regge }}^{(I)}(v, t) d v=\int_{v_{1}}^{v_{2}} v^{-n} \operatorname{Im} T_{\mathrm{t}, \text { Hadrons }}^{(I)}(v, t) d v,
$$


with $v=\frac{s-u}{2}=\frac{2 s+t-4 m_{\pi}^{2}}{2}$ and $s, t, u$ the standard Mandelstam variables. In $\pi \pi$ scattering, the relations between the $t$ - and $s$-channel amplitudes with definite isospin $I$ are [ 6 ]

$$
\begin{aligned}
& T_{\mathrm{t}}^{(0)}(s, t)=\frac{1}{3} T_{\mathrm{s}}^{(0)}(s, t)+T_{\mathrm{s}}^{(1)}(s, t)+\frac{5}{3} T_{\mathrm{s}}^{(2)}(s, t), \\
& T_{\mathrm{t}}^{(1)}(s, t)=\frac{1}{3} T_{\mathrm{s}}^{(0)}(s, t)+\frac{1}{2} T_{\mathrm{s}}^{(1)}(s, t)-\frac{5}{6} T_{\mathrm{s}}^{(2)}(s, t), \\
& T_{\mathrm{t}}^{(2)}(s, t)=\frac{1}{3} T_{\mathrm{s}}^{(0)}(s, t)-\frac{1}{2} T_{\mathrm{s}}^{(1)}(s, t)+\frac{1}{6} T_{\mathrm{s}}^{(2)}(s, t),
\end{aligned}
$$

where the subscript of $T$ labels the $t$ - or $s$-channel and the superscript stands for the isospin $I$. The left hand side of Eq. ([2.2) can be evaluated in Regge theory. The explicit expressions and results can be found in Ref. [W] and references therein. For the right hand side of Eq. (2.2), we decompose the isospin amplitudes into a sum of partial wave amplitudes

$$
\operatorname{Im} T_{\mathrm{s}}^{(I)}(v, t)=\sum_{J}(2 J+1) \operatorname{Im} T^{I J}(s) P_{J}\left(z_{s}\right),
$$

with $z_{s}=1+2 t /\left(s-4 m_{\pi}^{2}\right)$ the cosine of the scattering angle in the $s$-channel center of mass frame

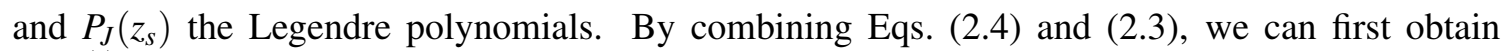
$\operatorname{Im} T_{t, \text { Hadrons }}^{(I)}(v, t)$ and then compare the hadronic contributions with those from Regge theory.

One expects that the "averaging" in Eq. (2.2) should start to work at least for one resonance tower and this means the integration range $v_{2}-v_{1}$ should be set as a multiple of $1 \mathrm{GeV}^{2}$. In this work we are interested in the energy region below $2 \mathrm{GeV}^{2}$. To proceed the discussion, it is advisory to consider the following ratios to cancel the uncertainties caused by the Regge couplings [च, []]

$$
\begin{aligned}
R_{n}^{I} & =\frac{\int_{v_{1}}^{v_{2}} v^{-n} \operatorname{Im} T_{\mathrm{t}}^{(I)}(v, t) d v}{\int_{v_{1}}^{v_{3}} v^{-n} \operatorname{Im} T_{\mathrm{t}}^{(I)}(v, t) d v}, \\
F_{n}^{I I^{\prime}} & =\frac{\int_{v_{1}}^{v_{\max }} v^{-n} \operatorname{Im} T_{\mathrm{t}}^{(I)}(v, t) d v}{\int_{v_{1}}^{v_{\max }} v^{-n} \operatorname{Im} T_{\mathrm{t}}^{\left(I^{\prime}\right)}(v, t) d v},
\end{aligned}
$$

with $v_{1}$ the $\pi \pi$ threshold, $v_{2}=1 \mathrm{GeV}^{2}, v_{3}=2 \mathrm{GeV}^{2}$ and $v_{\max }=2 \mathrm{GeV}^{2}$ in later discussions.

\section{Discussions}

We fit the unknown parameters in our theory to a large amount of experimental data, consisting of phase shifts and inelasticities of $\pi \pi \rightarrow \pi \pi(K \bar{K})$ and $\pi K \rightarrow \pi K$ scattering, with different isospin and angular momentum numbers, and also the invariant mass distribution of the $\pi \eta$ system [ [U, []]. The fit quality is fairly good and with the fitted parameters we obtain seven scalar and three vector resonances from our unitarized scattering amplitudes in the complex energy plane: $f_{0}(600)$, $f_{0}(980), f_{0}(1370), K_{0}^{*}(800), K_{0}^{*}(1430), a_{0}(980), a_{0}(1450), \rho(770), K^{*}(892)$ and $\phi(1020)$. Their masses and widths agree well with the PDG values. In addition, we also calculate the coupling strengths of the resonances to the pseudo-Goldstone boson pairs. This comprises the couplings of the $f_{0}$ resonances to $\pi \pi, K \bar{K}, \eta \eta, \eta \eta^{\prime}$ and $\eta^{\prime} \eta^{\prime}$, the couplings of $K_{0}^{*}$ to $K \pi, K \eta$ and $K \eta^{\prime}$, the couplings of $a_{0}$ resonances to $\pi \eta, K \bar{K}$ and $\pi \eta^{\prime}$ and also the relevant coupling strengths for 


\begin{tabular}{|l|l|ll|ll|ll|}
\hline & $\mathrm{n}$ & $R_{n}^{0}$ & $R_{n}^{0}$ & $R_{n}^{1}$ & $R_{n}^{1}$ & $F_{n}^{21}$ & $F_{n}^{21}$ \\
& $t=t_{\text {th }}$ & $t=0$ & $t=t_{\text {th }}$ & $t=0$ & $t=t_{\text {th }}$ & $t=0$ \\
\hline \hline Regge & 0 & 0.225 & 0.233 & 0.325 & 0.353 & $\sim 0$ & $\sim 0$ \\
& 1 & 0.425 & 0.452 & 0.578 & 0.642 & $\sim 0$ & $\sim 0$ \\
& 2 & 0.705 & 0.765 & 0.839 & 0.908 & $\sim 0$ & $\sim 0$ \\
& 3 & 0.916 & 0.958 & 0.966 & 0.990 & $\sim 0$ & $\sim 0$ \\
\hline Ours & 0 & 0.669 & 0.628 & 0.836 & 0.817 & -0.113 & 0.040 \\
$S+P$ & 1 & 0.837 & 0.812 & 0.919 & 0.908 & -0.230 & -0.087 \\
Waves & 2 & 0.934 & 0.924 & 0.966 & 0.962 & -0.129 & 0.028 \\
& 3 & 0.979 & 0.976 & 0.989 & 0.988 & 0.169 & 0.345 \\
\hline Ours & 0 & 0.410 & 0.400 & 0.453 & 0.468 & 0.531 & 0.587 \\
$S+P+D$ & 1 & 0.653 & 0.643 & 0.694 & 0.706 & 0.154 & 0.236 \\
Waves & 2 & 0.850 & 0.844 & 0.875 & 0.882 & 0.027 & 0.155 \\
& 3 & 0.954 & 0.953 & 0.965 & 0.968 & 0.225 & 0.388 \\
\hline \hline
\end{tabular}

Table 1: Values for $R_{n}^{I}$ and $F_{n}^{I I^{\prime}}$. Results by using two values of $t, 0$ and $t_{\text {th }}=4 m_{\pi}^{2}$, are shown in the table.

the vector resonances. One important lesson we learn is that the $f_{0}(600)$ resonance is marginally coupled to $\eta$ and $\eta^{\prime}$ mesons, indicating its insensitivity to the $\eta$ and $\eta^{\prime}$ dynamics. The explicit numbers can be found in Refs. [प, ]].

Now, it is interesting to explore the scattering amplitudes, which contain the relevant resonances, in finite energy sum rules. We compare the results from Regge theory and the unitarized $U(3) \chi \mathrm{PT}$ amplitudes in Table $\mathrm{W}$. When considering the $D$-wave contributions, we include the tensor resonances in meson-meson scattering following the framework in Ref. [ए2]. The first lesson we can learn from the numbers in Table $\square$ is that the results are stable when switching from $t=0$ to $t=4 m_{\pi}^{2}$. Next, let us focus on the ratios $R_{n}^{I_{\mathrm{t}}}$ with $I_{\mathrm{t}}=0,1$ and our current results qualitatively agree with the conclusions in Ref. [प]]. The semi-local duality with $n=3$ can be well satisfied by just $S$ - and $P$-waves, while the fulfillment for the $n=2$ case is marginal. About the cases with smaller values of $n$, higher partial waves and cut-offs are needed in order to fulfill the semi-local duality. As one can see in Table W, the inclusion of the $D$-waves clearly improves the situations for $n=0,1$.

Concerning the ratios $F_{n}^{21}$ with the isotensor amplitude involved, we observe different results. Due to the suppression of the Regge exchanges in $I_{\mathrm{t}}=2$ amplitude, the Regge theory predicts almost vanishing values for $F_{n}^{21}$. From Eq. (2.3]), one can simply conclude that if the $P$ - $(S-)$ wave contributions are dropped the ratios of $F_{n}^{21}$ should approach to $+1(-1)$, which can be viewed as the criteria to claim the extreme violation of semi-local duality. By only considering the $S$ - and $P$-waves, we find that semi-local duality is well satisfied for all values of $n$ and there is no clear sign that it is better satisfied for a specific value of $n$, contrary to the situations of $R_{n}^{I}$. While the introduction of the $D$-waves, instead of improving the situation, deteriorates the fulfillment of the 
semi-local duality for $n=0$.

All the discussions above are made in the physical situation, i.e. at $N_{C}=3$. It is interesting to investigate the fulfillments of semi-local duality by extrapolating the values of $N_{C}$ to larger numbers []]. In order to perform this study, we need to know the $N_{C}$ scaling of the parameters in our theory. For the $N_{C}$ scaling at leading order, it is known without any ambiguity, which can be found in Refs. [四, [0]. While if one wants to consider the sub-leading order $N_{C}$ scaling, extra assumptions need to be provided. In order to estimate the uncertainties of the sub-leading order effects, we propose four different scenarios to discuss semi-local duality. It is interesting to point out that semi-local duality indeed can distinguish different scenarios and hence provides us a way to constrain the $N_{C}$ evolution of parameters []. This also gives us a valuable onset to study the $N_{C}$

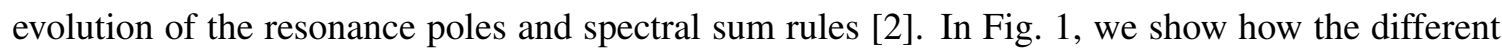
scenarios can be distinguished in the study of the ratios $F_{n}^{21}$. Taking into account the criteria we mentioned above, it is easy to conclude that Scenario 3 is the best one to satisfy semi-local duality. In Scenario 4, we take into account the $D$-wave contributions, in addition to the $S$ - and $P$-waves that are included in Scenarios 1-3. The reason why Scenario 4 is disfavoured is due to the fact that the $D$-wave overbalances the vector resonance contributions and hence it leads to too large values for the $n=0$ case. In the physical situation, as we mentioned before, the semi-local duality can be well satisfied by the $S$ - and $P$-waves, mainly due to the cancellations of the $f_{0}(600)$ and $\rho(770)$ in the amplitudes. At large values of $N_{C}$, although the $f_{0}(600)$ resonance fades away in the complex energy plane and hence, barely contributes to the amplitudes [ [ [ []], another scalar strength survives and behaves like a standard $q \bar{q}$ resonance. The latter one corresponds to the singlet scalar $S_{1}$ which is part of the $f_{0}(980)$ for $N_{C}=3$. This is a crucial source to oppose the $\rho(770)$ contribution and hence guarantees the fulfillment of semi-local duality at large values of $N_{C}$.
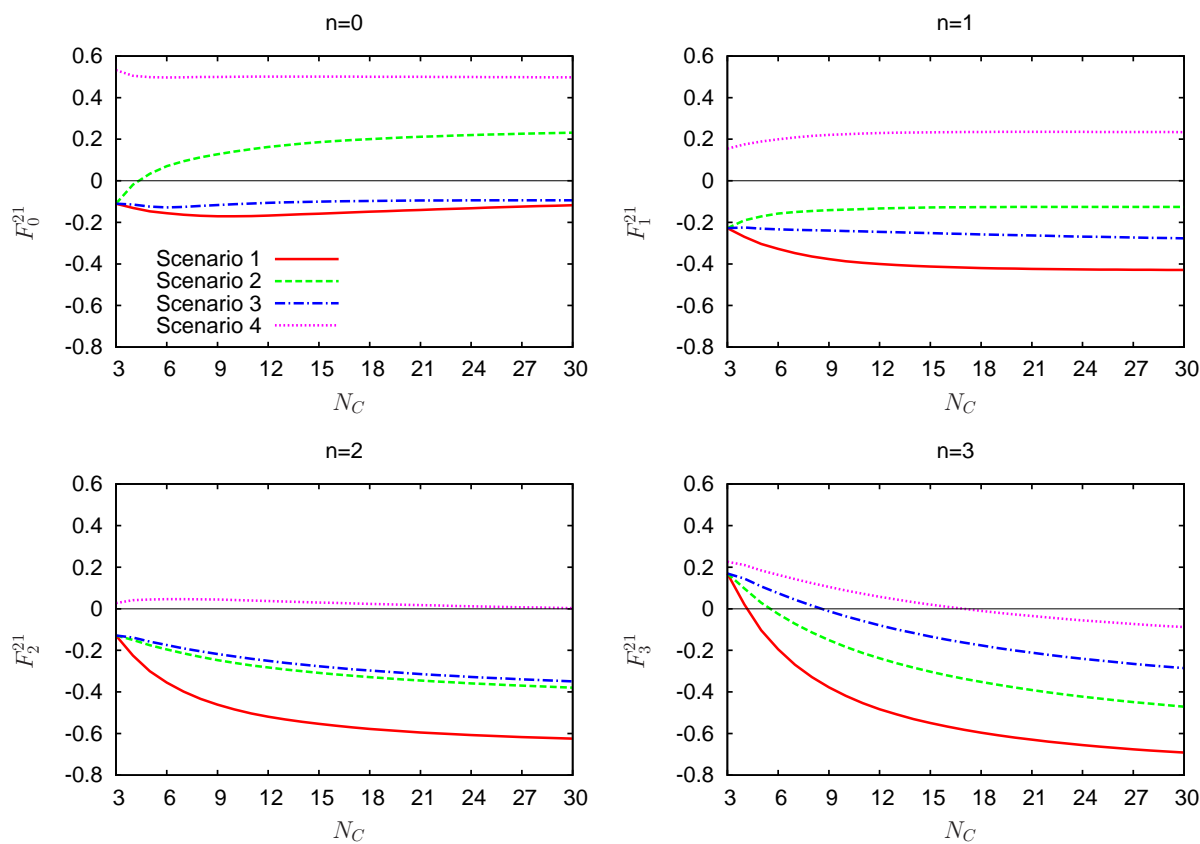

Figure 1: $F_{n}^{21}\left(t=4 m_{\pi}^{2}\right)$. Detailed definitions for the different scenarios can be found in Ref. [వ]]. 


\section{Acknowledgements}

This work is partially funded by the grants MEC FPA2010-17806 and the Fundación Séneca 11871/PI/09. We also thank the financial support from the BMBF grant 06BN411, the EU-Research Infrastructure Integrating Activity "Study of Strongly Interacting Matter" (HadronPhysics2, grant No. 227431) under the Seventh Framework Program of EU and the Consolider-Ingenio 2010 Programme CPAN (CSD2007-00042). Z.H.G. acknowledges CPAN postdoc contract in the Universidad de Murcia and financial support from the grants National Natural Science Foundation of China (NSFC) under contract No. 11105038, Natural Science Foundation of Hebei Province with contract No. A2011205093 and Doctor Foundation of Hebei Normal University with contract No. L2010B04.

\section{References}

[1] Z.-H. Guo and J.A. Oller, Phys. Rev. D 84, 034005 (2011).

[2] Z.-H. Guo, J. A. Oller and J. Ruiz de Elvira, Phys. Lett. B 712, 407 (2012); Z.-H. Guo, J. A. Oller and J. Ruiz de Elvira, arXiv:1206.4163 [hep-ph].

[3] J. Oller and E. Oset, Nucl. Phys. A 620, 438 (1997); (E)-ibid A 652, 407 (1999); R. Kaminski, J. R. Peláez and F. J. Ynduráin, Phys. Rev. D 77, 054015 (2008); R. Garcia-Martin, et al., Phys. Rev. D 83, 074004 (2011); I. Caprini, G. Colangelo and H. Leutwyler, Phys. Rev. Lett. 96, 132001 (2006); S. Descotes-Genon and B. Moussallam, Eur. Phys. J. C 48, 553 (2006); Z. Xiao and H. Q. Zheng, Nucl. Phys. A 695, 273 (2001); H. Q. Zheng, et al., Nucl. Phys. A 733, 235 (2004); Z. -Y. Zhou and Z. Xiao, Phys. Rev. D 83, 014010 (2011); J. Nieves, A. Pich and E. Ruiz Arriola, Phys. Rev. D 84, 096002 (2011); M. Albaladejo and J. A. Oller, Phys. Rev. Lett. 101, 252002 (2008); H. -X. Chen, A. Hosaka and S. -L. Zhu, Phys. Rev. D 76, 094025 (2007); H. -X. Chen, A. Hosaka, H. Toki and S. -L. Zhu, Phys. Rev. D 81, 114034 (2010).

[4] J. A. Oller and E. Oset, Phys. Rev. D 60, 074023 (1999).

[5] K. Nakamura, et al., J. Phys. G 37, 075021 (2010).

[6] P.D.B. Collins, An introduction to Regge theory and high energy physics. Cambridge University Press, Cambridge, 1977.

[7] P. Herrera-Siklody, J. I. Latorre, P. Pascual and J. Taron Nucl. Phys. B 497, 345 (1997).

[8] R. Kaiser and H. Leutwyler, Eur. Phys. J. C 17, 623 (2000).

[9] J. Gasser and H. Leutwyler, Nucl. Phys. B 250, 465 (1985).

[10] G. Ecker, J. Gasser, A. Pich and E. de Rafael, Nucl. Phys. B 321, 311 (1989).

[11] J. R. Peláez, M. R. Pennington, J. Ruiz de Elvira and D. J. Wilson, Phys. Rev. D 84, 096006 (2011).

[12] G. Ecker and C. Zauner, Eur. Phys. J. C 52, 315 (2007). 